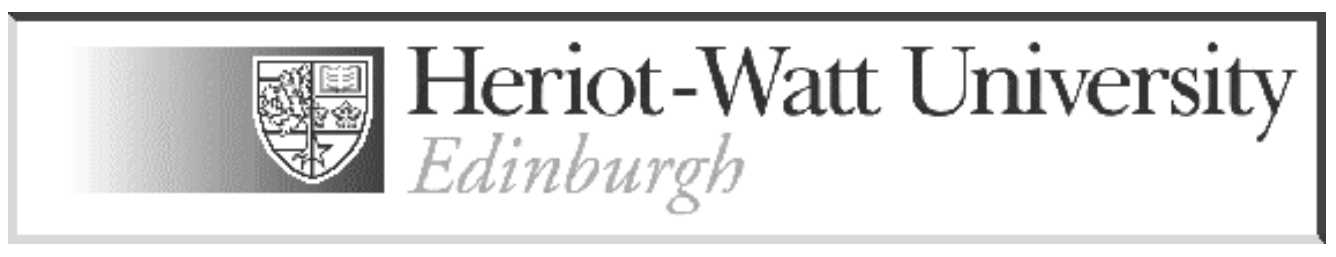

\title{
AN ANALYSIS OF THE LEVEL OF SECURITY PROVIDED BY THE MINIMUM FUNDING REQUIREMENT
}

\author{
BY ANDREW J.G. CAIRNS \\ HERIOT-WATT UNIVERSITY
}

\begin{abstract}
In this paper we investigate the effectiveness of the Minimum Funding Requirement (MFR) (pre-1999 version) under random future investment conditions. In particular, do the various liabilities calculated under the MFR deliver a suitable level of security to pension scheme members?

The paper considers active (and deferred) members and current pensioners separately.

For active members, Monte Carlo simulation is used to compare how the proceeds arising from investment of the pre-1999 MFR liability in an equity-backed personal pension would compare with the deferred pension promised by the scheme. It is found that while there may be the intended $50 \%$ chance that the personal pension has a better outcome there are very substantial downside risks for younger members. Investment strategies based upon index-linked gilts are shown to result, on average, in lower personal pensions but they provide an extremely effective means of limiting the downside risks.

For pensioners, Monte Carlo simulation is used to find the distribution of the initial amount of assets required to pay off precisely the pension liabilities as they arise. This investigation considers the effectiveness of the 12-year rule and different investment strategies. The 12-year rule in combination with an equivalent investment strategy is shown to provide reasonable security for a typical group of pensioners. However, if investments are restricted to an appropriate mixture of gilts then the level of security for pensioners is much increased. In particular, we investigate the use of value-at-risk reserves. It is found that $95 \%$ value-at-risk reserves are lower for a mixed conventional/index-linked gilts strategy than a mixed gilts/equity strategy.

It is argued, because of the high degree of downside risk, that there is no place in MFR calculations for high, anticipated equity returns. Instead rates of interest should be based solely upon fixed-interest and index-linked gilt yields.
\end{abstract}

\section{KEYWORDS}

MFR; simulation; downside risk; value-at-risk reserves; static hedging 


\section{INTRODUCTION}

In this paper we consider how effective the Minimum Funding Requirement is (as it exists at the end of 1998). In particular, do the liabilities calculated according to UK professional guidance (Guidance Note 27, Version 1.2, of the Manual of Actuarial Practice - MAP, 1998) and the principles underlying these calculations result in a satisfactory level of security for the members of a pension fund? This question is posed with reference to two groups of members: active members and current pensioners. (Deferred pensioners are treated in a very similar way to active members so we do not consider them here.)

The level of security is investigated by carrying out Monte Carlo simulations of the intended investment strategies.

In Section we consider active members. Here it is appropriate to consider individual members rather than the membership as a whole. The reason for this is that the pre-1999 MFR liability for each member would be treated as a transfer value into a personal pension fund if a scheme is wound up. We compare, therefore, the level of pension provided under an equivalent personal pension with the revalued deferred pension promised under the rules of the scheme.

In Section 3 we look at how much money is required to provide security for a group of pensioners. Here there are two approaches to the calculation of the MFR liability. On the one hand pensions could be transfered to an insurance company and the amounts guaranteed. This would have a specific cost after which there would be no uncertainty for the members. For a moderately sized scheme this represents the maximum amount required to provide total security. On the other hand a large scheme would, perhaps, wish to continue as a closed fund and run off the liabilities. For a large scheme it is intended that pension payments due in the next 12 years would be matched with appropriate gilts while more distant cashflows would be valued using equity rates of return (10\% per annum compared to the $8 \%$ assumption for gilts). This introduces the possiblity of investment risk and this is what we consider here.

This paper, as discussed above considers the MFR from the point of view of the members of a scheme. Greenwood and Keogh (1997) consider the effects of the MFR from the point of view of the employer and other interested parties. 


\section{ACTIVE MEMBERS AND DEFERRED PENSIONERS}

\subsection{The basis for calculation}

The logic behind the calculation of the MFR liability (in its pre-1999 form) for an individual member goes as follows:

- The member leaves behind a deferred pension which will include statutory revaluation in deferment. The amount of the liability will be invested in a single-premium personal pension. The premium will be invested $100 \%$ in equities up to 10 years before the retirement age (appropriate for the MFR) and shifting in equal instalments over the next 10 years into appropriate gilts.

- At retirement the personal pension account is used to purchase a pension. We will refer to the amount of this personal pension as $P P$ and to the amount of the revalued deferred pension as $D P$.

- These two pensions are calculated taking into account actual price inflation and investment returns over the period between exit from the scheme and retirement and annuity rates at the date of retirement.

- The amount of the MFR liability should be of a level which ensures that there is at least a $50 \%$ chance that the amount of the personal pension exceeds the amount of the revalued deferred pension (that is, $\operatorname{Pr}(P P>D P)=\operatorname{Pr}(P P / D P>1) \geq 0.5)$.

In this section, we consider the whole distribution of $P P / D P$ and not just $\operatorname{Pr}(P P / D P>1)$ or the median.

\subsection{Model scheme description}

Here we wish to focus on an analysis of the effects of economic and investment risk. We consider a scheme which has a very simple structure which provides single-life pensions only and no lump sum benefits. Pensions under the scheme and in the personal pension are assumed to increase fully in line with the Retail Prices Index (RPI).

In more detail, then, the details of the scheme are as follows:

- the normal retirement age is 65 ;

- no spouses pension is payable on death either in deferment or while the pension is in payment;

- $\quad$ on death in deferment a lump sum equal in value to the reserve will be paid out;

- $\quad$ no lump sum benefit is payable at retirement;

- $\quad$ in deferment pensions are revalued in line with RPI or at 5\% per annum over the full period of deferment if this is less;

- pensions in payment are payable annually in advance and receive full RPI increases.

The benefit on death before retirement is designed to be cost neutral relative to the mortality decrement. This means that we can assume that the rate of mortality in deferment is zero. Equivalently the personal pension should provide for a return of fund on death in deferment. In 
reality the benefits on death in deferment may be different causing small variations between the two vehicles of a type which we do not wish to investigate in this paper.

This simple scheme allows us to focus on the principle source of risk: the economic variables inflation, total returns on equities and gilts, annuity rates and market-value adjustors (MVA's).

A detailed set of formulae defining the calculation of the MFR liability for active members and deferred pensioners is given in Appendix A.

\subsection{Simulations}

The intention of the MFR basis is that the amount $(P P)$ of pension purchased at the date of retirement should have at least a $50 \%$ chance of exceeding the amount $(D P)$ of the revalued deferred pension had the member preserved their benefits in the scheme: that is,

$$
\operatorname{Pr}\left(\frac{P P}{D P}>1\right)=0.5
$$

For convenience, and in line with statistical convention, we will work with the cumulative distribution function $F(z)=\operatorname{Pr}(P P / D P \leq z)$. The MFR basis suggests, therefore that we should have $F(1)=\operatorname{Pr}(P P / D P \leq 1)=0.5$.

Simulations were carried out using the Wilkie model to consider the effectiveness of the MFR for individual members. In using the Wilkie model, the long-term medians were adjusted in a way to ensure that median returns on equities, fixed-interest gilts and index-linked gilts were consistent with the MFR assumptions. The volatilities in the Wilkie model were as in Wilkie's (1995) paper. The model was also adapted in a simple way to allow for investment in gilts with a term to maturity of between 1 and 30 years (the model covers only risk-free cash and irredeemable gilts).

Under the personal pension both equity and gilt investments were assumed to be subject to management charges of $1 \%$ per annum.

The simulations here assumed that pensions would increase in line with RPI. The pension increase assumption was therefore set appropriately. Under the PP the annuity factor applied at retirement was the present value of a single-life annuity valued using the market real-rate of interest on IL gilts.

For a range of ages 10,000 dependent simulations (see Appendix B) were carried out to consider the empirical distribution of the actual PP/DP. (Here the PP takes account of actual returns from age $x$ to age 65 and real yields at age 65 and the DP takes account of actual inflation between $x$ and 65 with reduction if the average rate of RPI exceeds 5\% per annum over this period.)

The results for different ages are all relatively similar. Here we discuss only $x=55$ and $x=25$ to bring out the main points. In this investigation we concentrate as much on the whole of the distribution of $P P / D P$ rather than on the median.

\section{$\mathbf{x}=\mathbf{5 5}$}

In Figure 1 we plot the empirical cumulative distribution of $P P / D P$ for $x=55$ at exit: that is, $F(z)=\operatorname{Pr}(P P / D P \leq z)$. There are three curves representing three different investment strategies: 


\section{$x=55$}

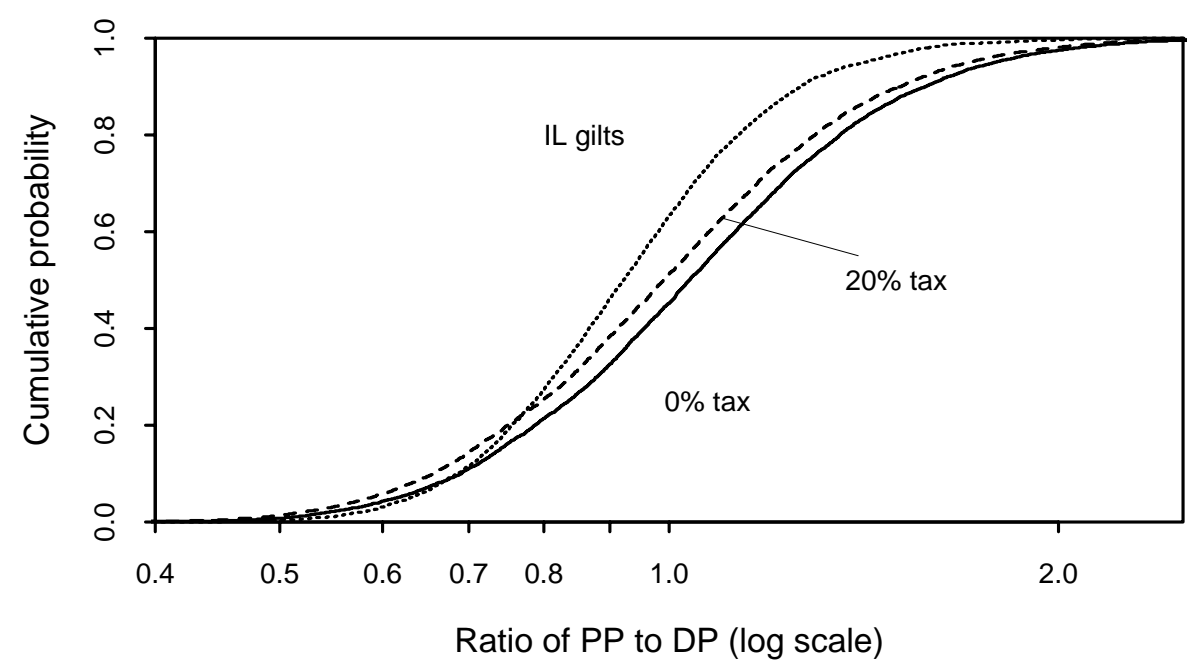

Figure 1: Empirical distribution of $P P / D P$ at retirement at age 65 for an active member now aged 55. Investment strategies: solid curve - equities shifting into gilts (with ACT credit); dashed curve - equities shifting into gilts (without ACT credit); dotted curve - $100 \%$ indexlinked gilts. 


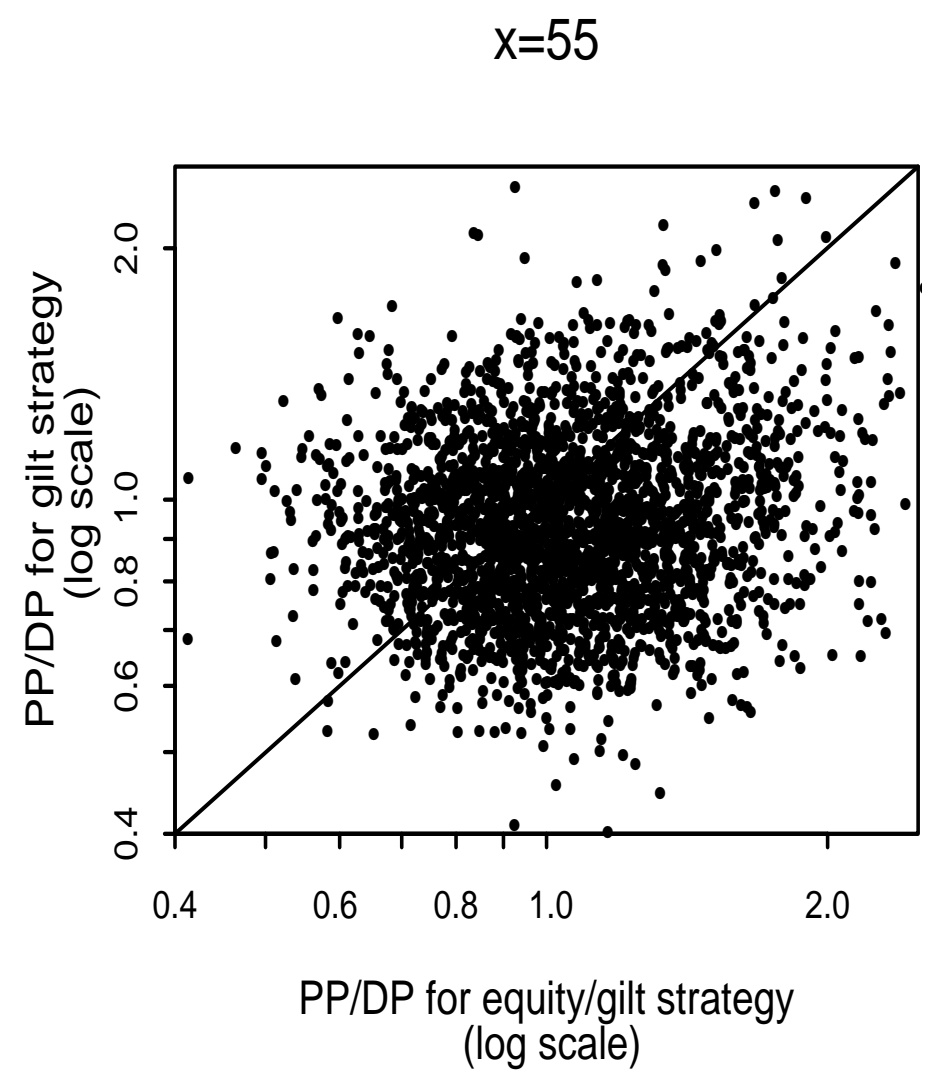

Figure 2: Comparison of the ratios $P P / D P$ for the equity/gilt strategy versus the index-linked gilt strategy. The diagonal line is ' $x=y$ '. About $38 \%$ of the points lie above this line representing the outcomes where the gilt strategy performs better.

- $\quad$ equities (with ACT credit) shifting into index-linked gilts;

- $\quad$ equities (without ACT credit) shifting into index-linked gilts;

- $\quad 100 \%$ index-linked gilts.

Under each strategy we used the same simulated investment data under the Wilkie model. From consideration if Figure 1 we note the following points:

- ACT credit removal has the effect of shifting the distribution a bit to the left. There is no effect on the spread.

- The median in the 0\%-tax equity/gilt case is slightly greater than 1 . The Wilkie model parameters could be adjusted further to ensure that $\operatorname{Pr}(P P / D P \leq 1)$ is precisely equal to 0.5. The need for additional adjustments (over and above those made earlier in Section ) would arise because of the non-linear effects of LPI in deferment. The change required to 
the parameter values would be very small but it would make the median rates of return on the various assets slightly inconsistent with the MFR basis.

- Even with only 10 years to retirement there are significant downside risks for the personal pensioner. For example, there is a $16 \%$ chance under the equity-based strategy that the PP is less than $75 \%$ of the DP.

- Conversely, there is a significant potential on the upside. For example, there is a $12 \%$ chance under the equity strategy that the ratio exceeds 1.5 .

- Investing $100 \%$ in index-linked gilts reduces significantly the spread of the distribution. Since gilts produce lower returns than equities in the long run the curve is also shifted to the left.

The main sources of uncertainty under this gilt-based strategy here are

- the equity MVA at the time of wind-up;

- $\quad$ the difference between RPI and LPI between ages 55 and 65.

- The cumulative distribution functions for the equity ( $0 \%$ tax) and gilt strategies cross over when the $P P / D P$ ratio is 0.67 . At this level both strategies have a probability of about $8 \%$ of a worse outcome. Below this cross-over point we can see that the worst $8 \%$ of cases under the equity based strategy are worse than the worst $8 \%$ of outcomes under the gilt strategy.

Furthermore we can note that in $38 \%$ of outcomes the gilt strategy actually performs better than the equity strategy (see Figure 2). This is a reflection of the relatively low degree of correlation between equity and gilt returns. Over a 10-year period the correlation between total returns on the equity/gilt strategy and the gilt strategy is 0.61 . Thus, even though the mean return on the latter was $2 \%$ per annum lower it still outperformed on $38 \%$ of occasions due to the high levels of variability. The one consequence of this is that it demonstrates how much the personal pensions of two otherwise identical members can differ by if they adopt different investment strategies.

If equities turn out to give a higher risk premium than $2 \%$ then this $38 \%$ would be reduced significantly.

As an alternative to the current MFR basis a purer form of market valuation was considered. Liabilities were discounted at gilt rates of return throughout and the gilt MVA was applied rather than the equity MVA. After transfer and prior to retirement assets were invested either wholly in index-linked gilts or $75 \%$ in index-linked and $25 \%$ in fixed-interest gilts. The results of this investigation are plotted in Figure 3 along with the result for the old basis with an index-linked investment strategy.

The obvious difference is that there is a general shift to the right. This is caused by the change in in the valuation rate of interest.

The change in basis also demonstrates quite how disruptive the application of the equity MVA is given the mismatch with an index-linked gilt investment strategy. Closer inspection of Figure 3 shows some differences between the different investment strategies. The 75/25 strategy gives a significantly lower standard deviation. On the other hand the 75/25 strategy has a fatter left hand tail which indicates a higher degree of downside risk. This gives us an example, then, of a simple static hedge. 
$x=55$

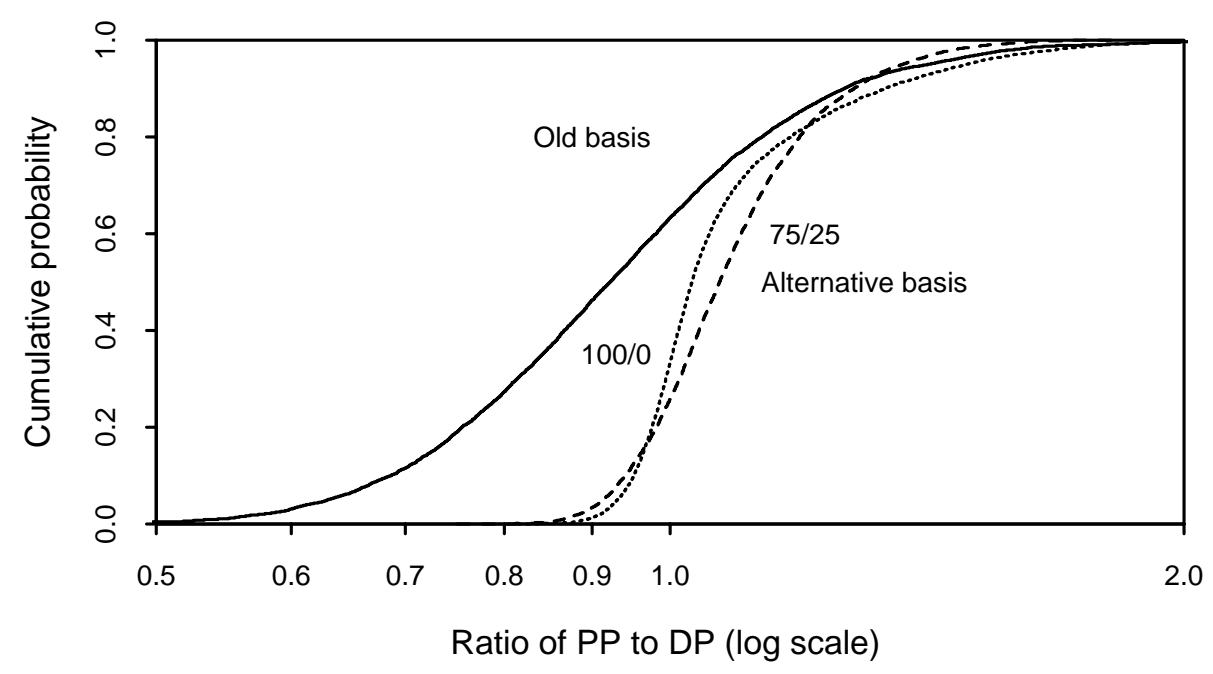

Figure 3: Empirical distribution of $P P / D P$ at retirement at age 65 for an active member now aged 55. Investment strategies: solid curve - old (current) MFR basis, investment $100 \%$ in index-linked gilts; dotted curve - alternative basis, $100 \%$ index-linked gilts; dashed curve alternative basis, $75 \%$ index-linked gilts, $25 \%$ fixed interest. 


\section{$x=25$}

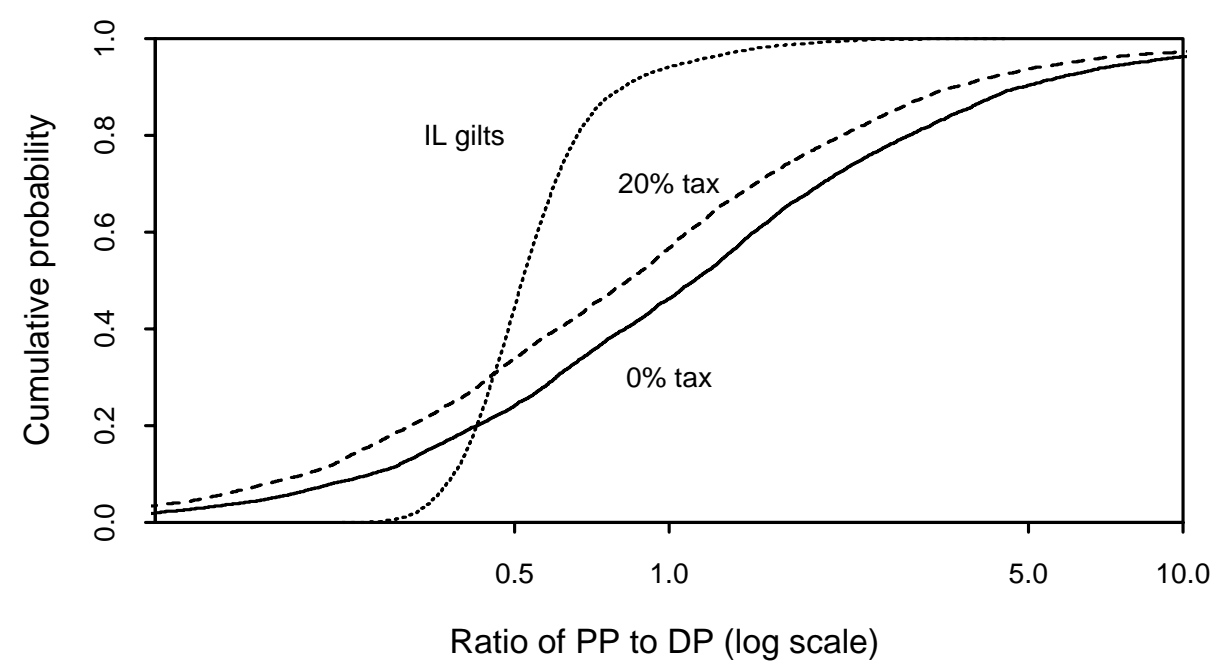

Figure 4: Empirical distribution of $P P / D P$ at retirement at age 65 for an active member now aged 25. Investment strategies: solid curve - equities shifting into gilts (with ACT credit); dashed curve - equities shifting into gilts (without ACT credit); dotted curve $-100 \%$ indexlinked gilts.

In either case the upper tail typically corresponds to outcomes where there was a relatively high level of inflation over the 10 years resulting in the implementation of the LPI limit. The lower tail outcomes arise because the valuation method is not a precise market-value and because of a degree of mismatching.

The level of risk could be reduced further if a more precise market value of liabilities could be calculated and if a dynamic hedging strategy using index-linked and fixed-interest gilts was used (that is, an asset allocation strategy which shifts money between different asset classes over time in reaction to changes in market conditions such as interest rates and inflation and due to the reducing time horizon).

$$
\mathbf{x}=\mathbf{2 5}
$$

The corresponding results for an member aged 25 at exit are plotted in Figures 4, 5 and 6. These should be compared with the corresponding Figures 1, 2 and 3 for $x=55$.

Here we see a much more dramatic picture.

- The effect of ACT credit removal is larger due to the longer period of investment in equities (that is, the shift to the left when we go from $0 \%$ to $20 \%$ tax is much greater in Figure 4 than in Figure 1.

- The equity based strategy (despite the switch into gilts after the personal pensionholder reaches age 55) is extremely risky:

- The median ratio is about right. 


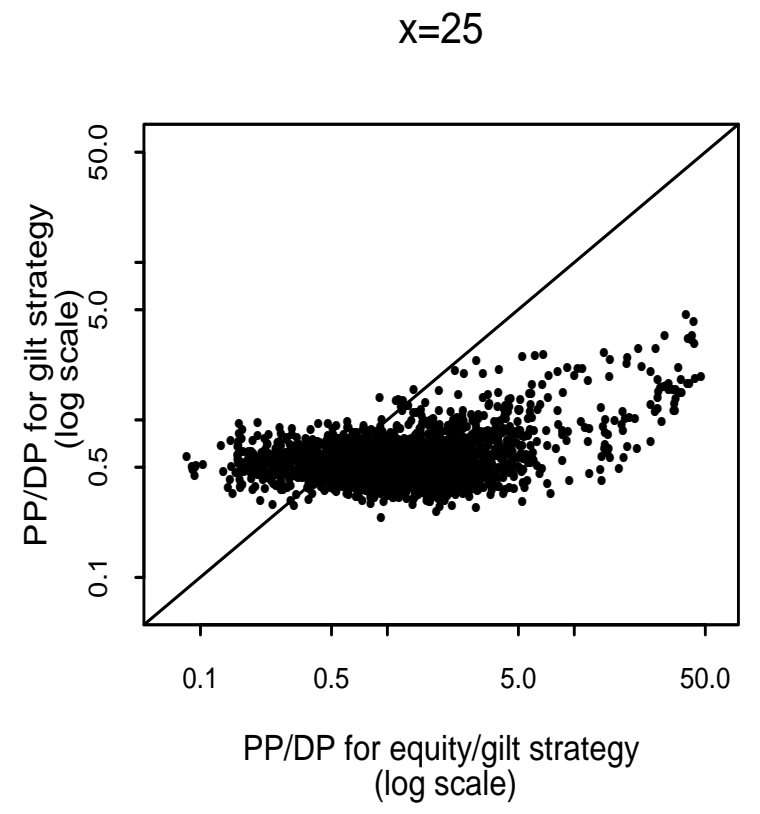

Figure 5: Comparison of the ratios $P P / D P$ for the equity/gilt strategy versus the index-linked gilt strategy. The diagonal line is ' $x=y$ '. About $32.5 \%$ of the points lie above this line representing the outcomes where the gilt strategy performs better.

- There is a good chance that the PP does spectacularly well relative to the DP (much more so than for the 55-year-old member).

- The PP can also do spectacularly badly relative to the DP. For example, there is a $24 \%$ chance that the PP is less than $50 \%$ of the DP. The downside risks here are much bigger than those for the 55-year-old.

- There is a reasonably strong correlation between the final ratio $(P P / D P)$ and average inflation over the period up to retirement with the lowest ratios corresponding to low inflation scenarios.

- A 100\% IL strategy performs much worse on average than the equity-based PP (the madian ratio is about 0.5 ). However, under either strategy the chances that the PP is less than about $42 \%$ of the DP are about the same (20\%). Below this level the IL strategy is far superior. Furthermore, because of the lack of correlation between equity and index-linked returns (see Figure 5), the index-linked strategy outperforms the equity strategy about $25 \%$ of the time.

- As with age 55 the risks under the $100 \%$ IL strategy are mainly due to the equity MVA at wind up and (less so) the difference between RPI and LPI up to retirement: even over a 40year period there is a significant chance that average RPI exceeds 5\% per annum. Most of the upper tail in this distribution corresponds to outcomes where average RPI exceeds $5 \%$. This is more evident from Figure 6 where the effect of the equity MVA has been removed. 


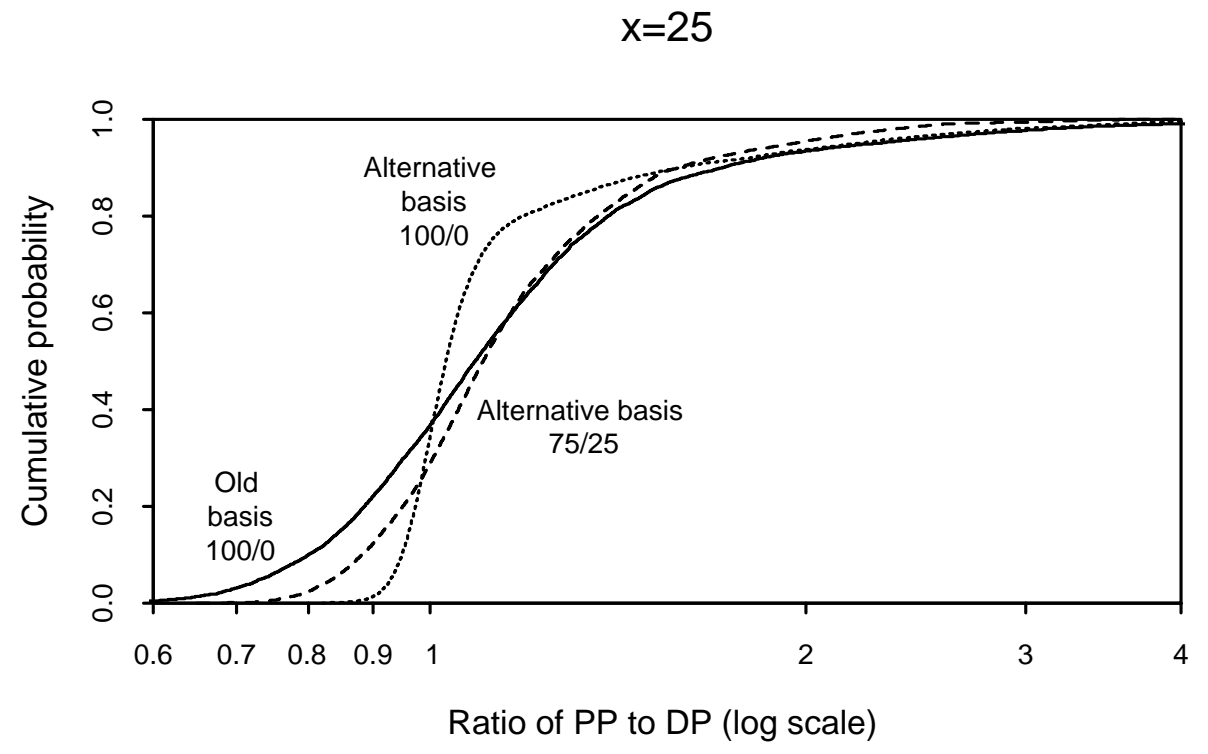

Figure 6: Empirical distribution of $P P / D P$ at retirement at age 65 for an active member now aged 25. Investment strategies: solid curve - old (current) MFR basis (but allowing for gilt returns rather than equity returns), investment $100 \%$ in index-linked gilts; dotted curve - alternative basis, $100 \%$ index-linked gilts; dashed curve - alternative basis, $75 \%$ index-linked gilts, $25 \%$ fixed interest. 
- From Figure 5 we can see that a simple static strategy investing in gilts and using giltbased MVA's rather than equity-based MVA's (but investing in gilts) reduces the level of downside risk for young members by a very significant margin.

These comparisons raise some significant points.

First, there is a point of view which says that equities provide a good hedge against inflation risk. In the Wilkie model over 40 years there is some correlation between inflation and returns on equities:

- $\quad$ if we invest $100 \%$ in equities over the full 40-year period then the correlation between the return on equities and the increase in the retail prices index is approximately 0.13 ;

- if we follow the same investment strategy up to age 55 and then allow for a switch into index-linked gilts between ages 55 and 65 then the correlation between the return on the investment fund over the whole 40-year period and the increase in RPI increases to about 0.78

- $\quad$ if the investment strategy invests $100 \%$ in index-linked gilts over the whole 40-year period then the correlation between the return of the fund and RPI is about 0.99 .

Clearly the level of correlation between equities and inflation would need to be very significantly higher to reduce the level of downside risk to young members to acceptable levels. With a different model this may be possible but there is no empirical evidence to support the use of such a model. Conversely in some financial-economic models RPI and equity returns might be linked only through the risk-free rate of interest so that the level of correlation over time could be much less. In consequence we conclude that equities do not provide a suitable matching asset for deferred-pensioners' liabilities.

Second, we can reasonably ask whether or not the market value adjustment for equities is reasonable (for gilts the adjustment is clearly reasonable provided the duration of the liabilities is right). The Wilkie model was investigated to see how returns are affected by current dividend yield. A simple linear regression was carried out to investigate the link between current dividend yield and the total return on equities over the next $n$ years. For example, suppose current gross dividend yields are $3.25 \%$ rather than the long term average of $4.25 \%$. This would mean that the equity MVA would be 1.31 suggesting that over a period of years equities would lose about a total of $27 \%$ (that is, $\log 1.31$ ) of growth relative to a scenario starting with a dividend yield of $4.25 \%$. The linear regressions showed that the reduction in return would be about $10 \%$ in the first year, $27 \%$ over 4 years, and around $36 \%$ over 10 or more years. This suggests that the MVA is not severe enough at all ages where the full equity MVA is applied. The reason for the excess loss over the MVA is a result of lower dividend yields over the first few years before they regress back to 'normal' levels. In contrast, the MVA adjustment would work only if dividends reverted immediately to the long-term MFR assumption.

In other cases it can be argued that the MVA is too big an adjustment. For example, if dividend yields are low in anticipation of larger than average dividend increases then one could argue that the MVA makes too big an adjustment.

\subsection{Other ages}


Results are similar for other ages being a mixture of the observations for ages 25 and 55 with a relatively uniform transition between the two. Further analysis is, therefore, uninformative.

\subsection{Sources of difference between medians}

It has been remarked earlier that it is the aim of the MFR liability that the median of the distribution of the personal pension to deferred pension ratio should be equal to 1 . We have seen that, in general, the median is close to but not equal to 1. This can happen for a number of reasons. In some cases the size of the deviation depends upon the number of years to retirement. In other cases the distance of the median from 1 is relatively independent of age. We will consider next what these reasons are.

\subsubsection{Non-age-dependent factors}

A number of non-age-dependent factors were present potentially within these simulations:

- The mean annuity rate at retirement for the Personal Pension could differ from the assumption in the valuation basis when multiplied by the mean gilt MVA. Here, the mean annuity rate at retirement for the Personal Pension was 11.69. This compares with a factor of 11.7 under the MFR basis so that there was in fact no significant deviation.

- If the mean equity MVA is above or below 1 then this will be a fixed source of profit/loss. Here, the mean equity MVA was 1.00 after the central dividend yield in the Wilkie model had been altered so there was again no effect.

Other factors may also arise in practice:

- $\quad$ There may be a difference between the LPI assumption made by the annuity provider and the basis.

- $\quad$ There may be a difference between the MFR mortality assumption and the mortality assumption made by the annuity provider.

- There may be small differences between the subsidiary benefits provided by the two options: for example, the benefit on death in retirement.

- $\quad$ The expense assumptions may differ in practice.

\subsubsection{Age-dependent factors}

- The median total return on equities (and to a lesser extent gilts) may differ from $10 \%(8 \%)$. The effect is magnified for younger members.

- The MFR basis uses the simple assumption that LPI in deferment will average out at $4 \%$. This is equal to the RPI assumption. Strictly the LPI assumption should be less than the RPI assumption to reflect the 5\% cap, but a true 3.8\% might, of course, be rounded to to $4 \%$ in the interests of simplicity. It is felt, though, that this assumption overstates the liability especially for older members and, perhaps, less so for younger members. There is a good 
chance that inflation over a period on $n$ years in a specific Wilkie scenario averages out at above $5 \%$ per annum. Even with $n=40$ there is a significant chance that this will happen (under the Wilkie model used here: 27\%). Furthermore instead of a fixed assumption it would be reasonable to take current inflation into account.

Of course, taking account of these differences would introduce added complexity to the basis.

\subsection{Further comment}

We can also investigate how members of the same cohort but of different ages compare when they retire. For example, suppose a scheme winds up in year 0 forcing members down the equity based PP route. Let us compare two members one aged 35 with 10 years past service and one aged 45 with 20 years past service. Furthermore, their salaries are such that the projected deferred pensions when both are in payment will be in the ratio two to one (in favour of the 45-year-old). These two members will retire 10 years apart but we can compare their PP/DP ratios. Since they left the scheme at the same time much of their PP investment experience will overlap so that we might expect that the two ratios will be highly correlated. This is indeed the case: the correlation coefficient is about 0.85 . However, this is with respect to ratios which can range approximately from 0.1 to 10 . Even with this high correlation coefficient we find that one PP/DP ratio can be more than 2 times the ratio for the other member. In particular there is a reasonable chance (in simulations this came out at about 7\%) that the 35-year-old ends up with a pension which is more than that of the 45 -year-old even though it was only projected to be a half.

This serves to illustrate the inequities which might arise when we compare members retiring at different times. The differences would be compounded if different members chose different pension providers or different investment vehicles.

\subsection{Deferred pensioners}

Deferred pensioners are subject to the same sorts of problems as active members, since they are treated identically to active members in MFR calculations, so we do not consider them here.

\section{Pensioners}

Here we considered a "model" set of pensioners in a mature scheme.

The basic scheme consisted of 25 members of various ages (details of these members are given in Appendix C) and each with a random future lifetime (different for each random investment scenario) determined with reference to PA(90)-2. Pensions were increased in line with LPI. Assets up to the MFR market-value of the liabilities arising in the first $n$ years were invested in index-linked gilts with a term of $T$ years to maturity. Any surplus assets were invested in equities (there is, therefore, never a negative holding of equities).

The value of $n$ was varied to alter the balance of assets invested in gilts. With $n=12$, initially $82 \%$ of the assets were invested in gilts. It was not felt necessary to consider the value 
of $n$ in relation to the $£ 100 \mathrm{M}$ rule. Instead we look at the effects of changing from $n=\infty$ to $n=12$ for a given scheme and we discuss under what circumstances this is justified from a probabilistic point of view. The figure of $£ 100 \mathrm{M}$ represents a perceived level at which it becomes economically more sensible to continue management of the pensioners in the scheme as a whole rather than switch their liabilities into an insurance company.

1000 independent Wilkie scenarios were generated (running up to the time of death of the last pensioner). In each scenario, where mortality is stochastic, the random future lifetimes of all individuals are independent and independent of the investment scenario. Under each scenario, $i$, an amount of assets, $F_{i}$, was calculated along the following lines:

- $\quad F_{i}$ would be invested in gilts and equities. The amount invested in gilts would be equal (as mentioned above) to the value of liabilities arising in the first $n$ years $\left(M V_{p 1}(n)\right)$ or the total amount of assets if this was less. Any surplus assets would be invested in equities.

- $\quad$ under the above investment strategy and with Wilkie scenario $i$ the amount $F_{i}$ would be sufficient to ensure that there was precisely enough money to pay off the benefits as they arose.

Each $F_{i}$ is the random present value as determined by the Wilkie scenario and the scenario generating the random future lifetimes.

The $F_{i}$ determine an empirical cumulative distribution function $\tilde{F}(z)$ (for example, see Figure 7). Suppose that we hold assets of $A$ at time 0 . Then $\tilde{F}(A)$ is an estimate of the probability that we will have sufficient assets to pay off the liabilities as they fall due.

We can consider the MFR liability in the context of this empirical distribution to determine its appropriateness (that is, the wider the spread of the distribution of the $F_{i}$ the less appropriate is the basis of the MFR calculation). Conversely we can use the empirical distribution to calculate value-at-risk reserves: that is, what is the reserve required to ensure that we have, say, a 95\% chance of having sufficient cash to pay for the promised benefits as they arise. This reserve is equal to $\tilde{F}^{-1}(0.95)$.

\subsection{Results}

- In the first two experiments (Figures 7 and 8) gilt-backed liabilities were invested entirely in 15-year index-linked bonds with a coupon rate of 3.85\%. (Note that in these figures and in Figure 9, a shift to the left of the cumulative distribution function, $F(z)$, represents an improved outlook. This is in contrast to those figures in Section 2 where a shift to the left - for example, from $0 \%$ to $20 \%$ tax - is bad.)

- The first experiment considered the effect of stochastic mortality. This involved three groups of simulations, each using the same 1000 Wilkie scenarios. In the first group the scheme started off with 25 pensioners. In the second group there were 100 pensioners (being 4 identical copies of the smaller scheme). In the third group the simulation used 25 members with deterministic mortality. The empirical distribution functions are plotted in Figure 7a (for comparison, present values with 100 members are divided by 4).

As we might expect there was hardly any movement in the medians of the three empirical distributions. However, the medians at about 6.09 are below the MFR reserve for this 


\section{stochastic vs deterministic mortality}
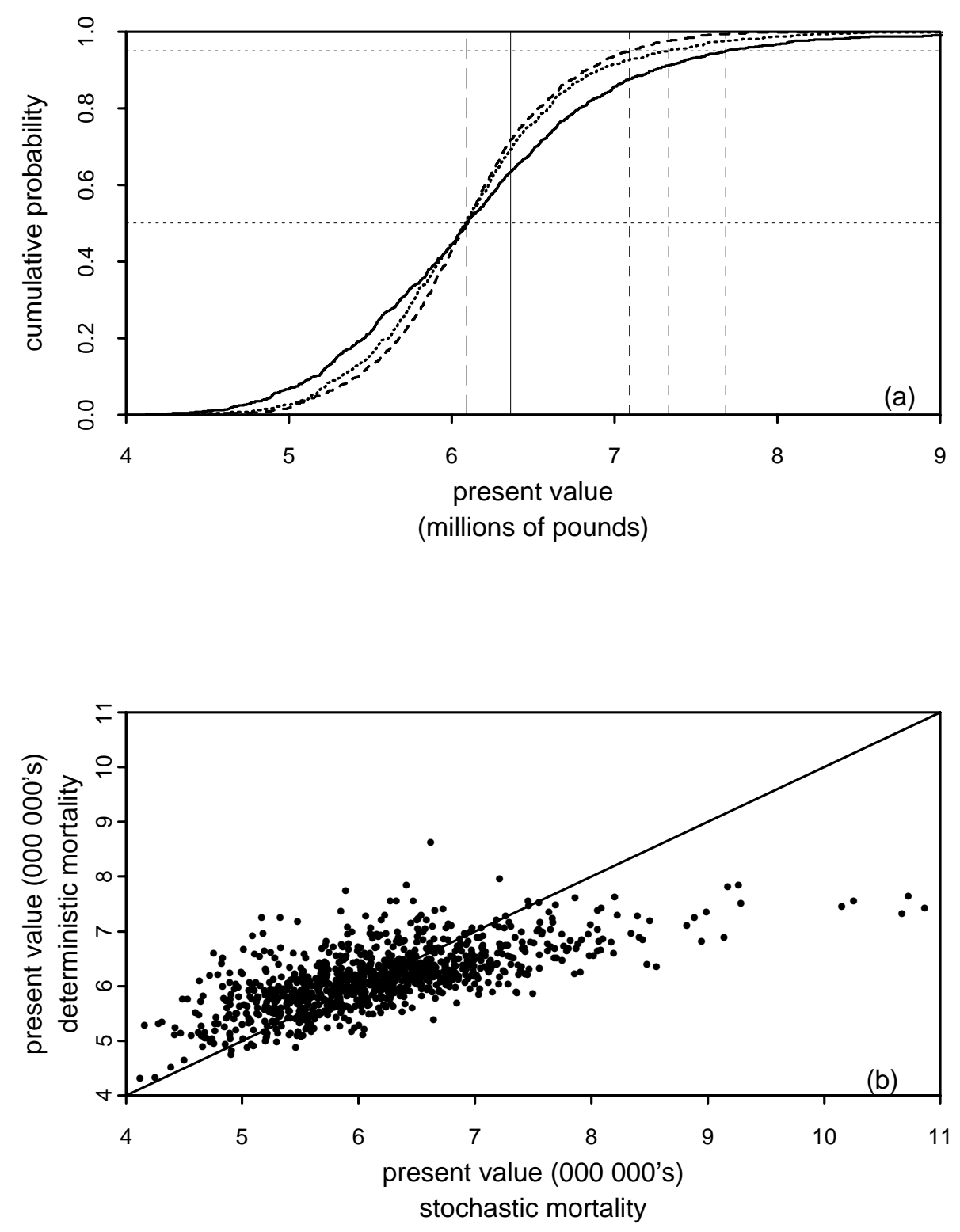

Figure 7: (a) Empirical cumulative distribution function, $F(z)$, for the random present value, $Z$, of the pensioners liability with $n=12$. Solid curve -25 members; dotted curve - 100 members (present value divided by 4); dashed curve - 25 members, deterministic mortality. Horizontal lines at the 50\% and 95\% level. Vertical lines at the median (long dashed line); MFR liability (solid); 95\% value-at-risk reserves (short-dashed). (b) Plot of the present value under stochastic against the present value under deterministic mortality for 1000 individual realisations of the Wilkie model. 


\section{gilt/equity vs gilt strategy}

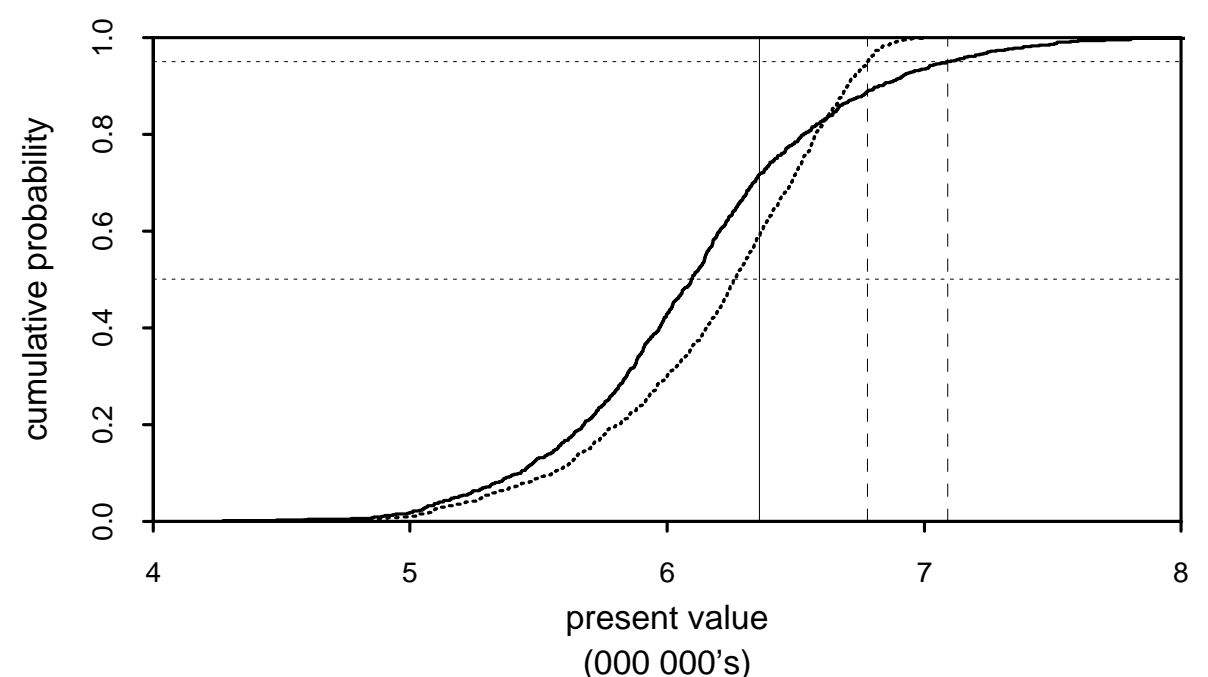

Figure 8: Empirical cumulative distribution function, $F(z)$, for the random present value, $Z$, of the pensioners liability with deterministic mortality. Solid-curve $-n=12$, gilt backed liabilities invested in 15-year IL gilts; dotted curve $-n=40$, gilt backed liabilities invested in IL gilts. Horizontal lines at the 50\% and 95\% level. Vertical lines at the MFR liability for $n=12$ (solid); and $95 \%$ value-at-risk reserves (short-dashed).

group which is 6.36. This suggests that the method of calculating the market value of the liabilities (that is, by taking the minimum of the reserves for $5 \%$ increases and $R P I-\frac{1}{2} \%$ increases) is too pessimistic (in the sense that the probability of a surplus is more than 0.5 ). On the other hand, we can look at the 95\% value-at-risk reserves. These are 7.68 with 25 members, 7.33 with 100 members (both with stochastic mortality) and 7.09 with 25 members and deterministic mortality. This gives us something of a feel for the effect of mortality risk on a small scheme. If we are interested only in the median as the MFR benchmark then clearly there is no justification for treating small and large schemes differently. On the other hand this is a reasonable thing to do if the value-at-risk approach is taken.

In some small schemes, total liabilities might be dominated rather more by a small number of very large pensions. In such cases the level of mortality risk would be higher.

In Figure $7 \mathrm{~b}$ we can compare the results for deterministic and stochastic mortality. In this graph the vertical placement of a point is due to economic factors, while the horizontal placement is due to the effects of stochastic mortality.

- The value of $n$ was varied (Figure 8) under the assumption of deterministic mortality. The shape of the curve for $n=12$ would give a typical picture for a large scheme with well over $£ 100 \mathrm{M}$ of pensioners liabilities. $n=\infty$ is the relevant value for a smaller scheme. (For computational convenience $n=40$ was used rather than $n=\infty$. This is acceptable 


\section{mixed gilt strategies}

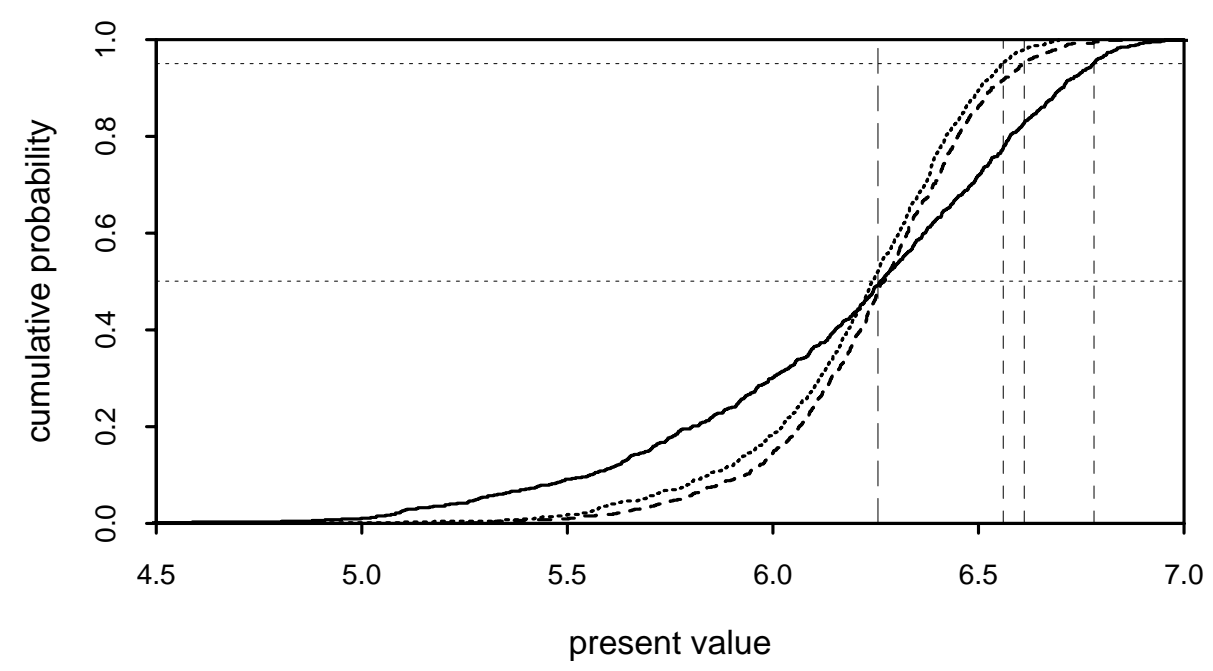

Figure 9: Empirical cumulative distribution function, $F(z)$, for the random present value, $Z$, of the pensioners liability with deterministic mortality and $n=40$. Solid-curve $-100 \%$ IL gilts; dotted curve $-75 \%$ IL gilts, $25 \%$ fixed interest gilts; dashed curve $-50 \% / 50 \%$.

given that the difference in present values as a result of the change is negligible.) Taking $n=40$ has the direct effect of adding about $5 \%$ to the current MFR liability (if we consider medians we get 6.26 against 6.09) given that gilts yield less on average than equities in the MFR basis. (This increase would be higher for a group of pensioners with a lower average age or with spouses pensions.) This increase is reversed if we consider $95 \%$ value-at-risk reserves which are 7.09 for $n=12$ and 6.78 for $n=40$ (that is, $11.5 \%$ and $6.6 \%$ above the usual MFR liability).

- There was very little difference in individual outcomes between investment in 2-year, 6year and 15-year 3.85\% IL gilts. In part this may be due to the Wilkie model in which the real-yield curve experiences only parallel shifts. This means that returns on IL gilts of different durations are very highly correlated giving the observation just noted.

- As an alternative to gilt investments entirely in index-linked stock we considered investing in a mixture of 15-year fixed-interest and index-linked gilts (Figure 9) and with no investment in equities (that is $n=40$ ). Three mixtures were considered: 100\% index-linked gilts and $0 \%$ fixed-interest; 75/25; and 50/50. As can be seen in Figure 9 the investment strategy has little effect on the median. There is, however, a significant effect on the $95 \%$ value-atrisk reserves. These are, respectively, $8.4 \%, 4.9 \%$ and $5.7 \%$ above the median. Conversely at the lower end of the distribution the tail was much thinner: that is, the potential for having a substantial surplus under the $75 / 25$ or $50 / 50$ strategies once all of the liabilities had been run off was much reduced.

It seems, therefore, that some investment in fixed interest and index-linked provided a more 


\section{inflation vs present value}

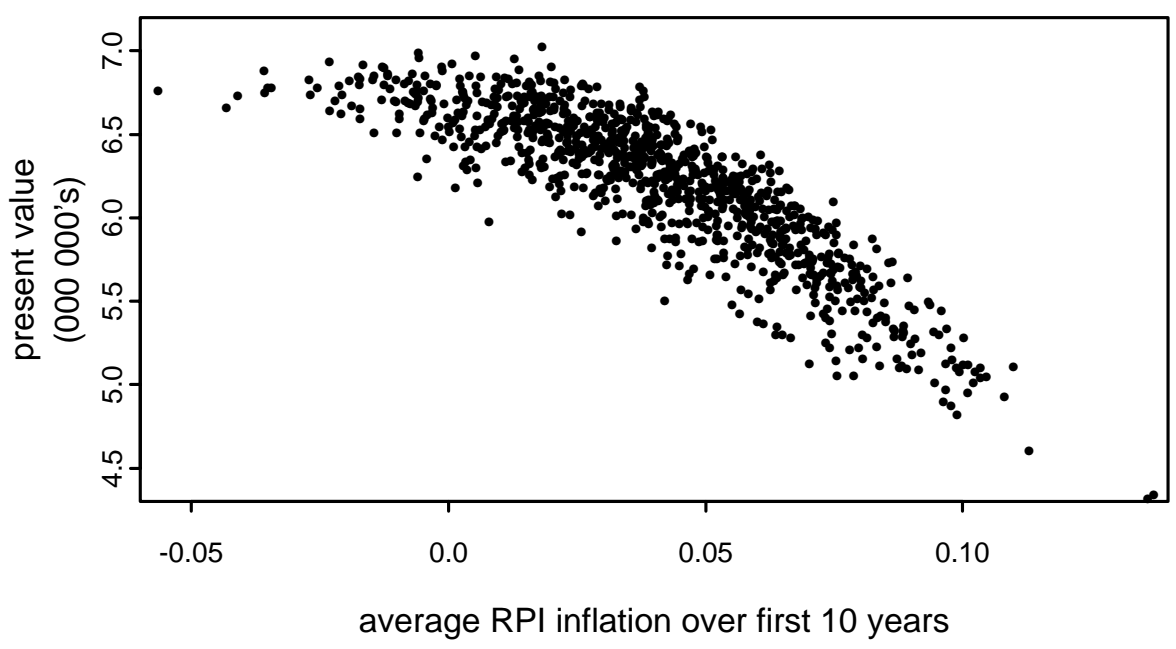

Figure 10: A demonstration of the strong correlation between the random present value and average RPI over the first 10 years. Investments are entirely in 15-year index-linked gilts.

effective hedge in an LPI environment. Clearly a more dynamic hedging strategy would be able to reduce the spread of the distribution almost to zero. Under such circumstances a 95\% reserve will be substantially less than an unhedged gilt/equity portfolio.

- In Figure 10 we have plotted the random present value $F_{i}$ against $R_{i}$ (average inflation over the first 10 years of simulation $i$ ) for $n=40$ and investment $100 \%$ in index-linked gilts. It can be seen that surplus is most likely to arise (that is, a low value of $F_{i}$ ) when average inflation is high. This is because the LPI cap is being applied more frequently while the index-linked investments are capable of supporting full RPI increases. This negative correlation is reduced when a mixture of fixed-interest and index-linked gilts are used and it becomes a positive correlation when less than $50 \%$ of the assets are invested in indexlinked gilts.

- In a scheme with a spouses pension the liabilities will have a longer duration resulting in a slightly bigger proportion of the fund invested in equities. This will increase the variance of the random present value of the benefits and, for example, increase the size of the $95 \%$ value-at-risk reserve.

- Overall the MFR calculation seems reasonable, mainly because the level of investment in gilts is already relatively high. The degree of uncertainty would be much reduced and the difference between the value-at-risk reserve and the MFR would be smaller if LPI-linked gilts were available or if the fund was to provide RPI rather than LPI annuities.

However, regardless of the size of the fund or of the membership, the MFR liability is far short of guaranteeing that the assets will be sufficient to cover the benefits as they arise (since the MFR is based upon the median present value). Since the median is not affected 
by fund size there is no justification for different treatment of small and large schemes. On the other hand, if a value-at-risk approach was adopted then equity easement might be suitable as an alternative to explicit reserving. Furthermore, when we consider the effects of stochastic mortality we see that differential treatment between large and small schemes is valid in the value-at-risk framework.

The arguments in favour of equity easement are:

1. In the event of wind-up, a small scheme would have to purchase annuities on the basis of gilt rates of interest only. A large scheme would find it more efficient to continue to run off the liabilities as a closed fund until the assets are insufficient to allow the fund to be managed in an economically viable fashion (that is, when management costs become to high and there are difficulties in achieving an adequate degree of diversification).

2. Equities will perform better than gilts in the long run allowing fund managers to switch into gilts at the right moment in the future, guaranteeing what remained of the promised pensions.

It is point 2 where this argument is flawed. There seems to be an implicit assumption that equities are guaranteed to outperform gilts by at least $2 \%$ per ammum on average over a number of years. This is a contradiction of the MFR principle for active members under which median outperformance is $2 \%$ per annum.

Suppose we accept that median outperformance of $2 \%$ per annum is the correct interpretation. Then this means that members in a large scheme applying equity easement have less secure benefits than members of a small scheme since large-scheme members are subject to substantial risks that their benefits will have to be reduced in the future should the scheme be wound up.

\section{Conclusions}

We will consider active/deferred members and pensioners in turn.

\subsection{Actives}

Anyone with expertise in pensions knows that the MFR (as it stood at the end of 1998) does not provide members with a guarantee. Instead it defines a 'reasonable' amount of money to be held in reserves which may, in the event, turn out to be insufficient. However, do members fully understand this difference? Furthermore, do members or the majority of professionals fully understand how seriously the personal pension vehicle can underperform relative to the deferred pension given up? Of course the personal pension can also do much better, but this misses the point: from the point of view of the member, underperformance is usually much more significant than outperformance.

It has been demonstrated in this paper that with a suitable MFR calculation and with a properly matched investment strategy the downside risks for a member can be reduced very significantly. 
It may be felt that the general level of the MFR on the current basis is about right from a political point of view. If this is the case would it not be preferrable to express the MFR liability as, say, $80 \%$ of the true market value of the liabilities. Such a value, ideally, is calculated using varying future market rates of interest (nominal and real) and inflation rates (for example, using the curves suggested by Feldman et al., 1998, and Cairns, 1998). This would provide much more of a minimum guarantee for members (albeit less than 100\%) and a greater degree of equity.

\subsection{Pensioners}

The calculation of the MFR liability for pensioners is based largely on gilt investments. As such the level of downside risk for this group is relatively small. Nevertheless, the level of risk to the pension scheme members can be reduced substantially if (a) there is no equity easement and (b) a suitable hedging strategy is employed.

As with active members, it would be appropriate (and less risky still) if a more precise market value of the liabilities could be established. For minimum risk this should be combined with a suitably matched portfolio or with suitable dynamic hedging in the case of LPI pensions. Given that the current MFR basis for pensioners is largely backed by gilts, this change would not increase the amount of the liability by a great deal.

\section{ACKNOWLEDGEMENTS}

An earlier version of this paper formed part of the author's contribution to the work of the MFR Change of Conditions Working Party. The opinions expressed in this paper are those of the author and not necessarily those of the working party.

The author would like to thank all of the members of the MFR Change of Conditions Working Party (in particular, Chris Long and Eddie Battersby) and two anonymous referees for their comments on earlier drafts of this paper. 


\section{REFERENCES}

CAIRns, A.J.G. (1998) Descriptive bond-yield and forward-rate models for the British government securities' market (with discussion). British Actuarial Journal 4, 265-321.

Feldman, K.S., Bergman, B., Cairns, A.J.G., Chaplin, G.B., Gwilt, G.D., LOCKYER, P.R., AND TURLEY, F.B. (1998) Report of the fixed interest working group. British Actuarial Journal 4, 213-263.

Greenwood, P.M., And Keogh, T.W. (1997) Pension funding and expensing in the minimum funding requirement environment (with discussion). British Actuarial Journal 3, 497-582.

MAP (1998) Manual of Actuarial Practice. Institute of Actuaries and Faculty of Actuaries.

WILKIE, A.D. (1995) More on a stochastic asset model for actuarial use (with discussion). British Actuarial Journal 1, 777-964.

\section{CONTACT ADDRESS}

Andrew J.G. Cairns, Department of Actuarial Mathematics and Statistics, Heriot-Watt University, Edinburgh, EH14 4AS, United Kingdom. E-mail: A.Cairns@ma.hw.ac.uk WWW: http://www.ma.hw.ac.uk/ andrewc/ 


\section{APPENDICES}

\section{APPENDIX A \\ MFR CALCULATIONS}

The liability for an active member aged $x$ exact is calculated as follows:

First we calculate the value of the discounted present value of the liability using long-term valuation assumptions.

$$
\begin{aligned}
P V_{a} & =\frac{N}{60} \times F P S \times(1+r e v)^{65-x} v_{i=9 \%}^{65-x} \ddot{a}_{65, i^{\prime}} \times(1+0.005 n) \\
\text { where } n & =\min (65-x, 10) \\
r e v & =0.04=\text { rate of revaluation in deferment }
\end{aligned}
$$

Here $\ddot{a}_{65, i^{\prime}}$ is the present value of a pension of 1 payable annually in advance and increasing at $4 \%$ per annum for full RPI increases (or $3.5 \%$ per annum as a proxy for LPI increases). Thus the net rate of interest is $i^{\prime}=1.08 / 1.04-1=0.03846$. The rate of interest in deferment of $9 \%$ is equal to the long term rate of return expected on equities (10\% per annum) less an allowance of $1 \%$ per annum for fund-management expenses.

We estimate the market value of the liability by applying an appropriate market value adjuster, taking account of the supposed mix of assets for each member (100\% equities for members 10 or more years from retirement).

$$
M V_{a}=\left(\frac{n}{10} \times M V A_{e q}+\frac{10-n}{10} \times M V A_{g}\right) \times P V_{a}
$$

where $M V A_{e q}=4.25 / D_{\text {all-share }}$

$D_{\text {all-share }}=$ gross dividend yield on the FT-actuaries all-share index

$$
M V A_{g}=c \cdot a_{\overline{15}, y}+v_{y}^{15}
$$

where

fixed interest:

$$
\begin{aligned}
c & =\text { coupon rate } \\
& =0.08 \\
y=y_{0} & =\text { annualised yield on } 15 \text {-year medium coupon gilts index }
\end{aligned}
$$

index-linked:

$$
\begin{aligned}
& c= 1.08 / 1.04-1=0.03846 \\
& y=y_{1}= \text { annualised real yield on the over-5-year index-linked gilts } \\
& \text { index (with a } 5 \% \text { inflation assumption) }
\end{aligned}
$$


The choice of gilt MVA depends upon what is regarded as the appropriate matching asset for the liabilities. In this study it is the index-linked MVA.

The liability for a deferred member aged $x$ who left the scheme at age $y$ exact (time $t-(x-y))$ with a preserved pension before revaluation of $D P$ is

$$
\begin{aligned}
P V_{d} & =(1+0.005 n) D P \min \left[\frac{R P I(t)}{R P I(t-x+y)} \times(1+r e v)^{(65-x)}, 1.05^{(65-y)}\right] v_{i=9 \%}^{65-x} \ddot{a}_{65, i^{\prime}} \\
M V_{d} & =\left(\frac{n}{10} \times M V A_{e q}+\frac{10-n}{10} \times M V A_{g}\right) \times P V_{d}
\end{aligned}
$$

Note that in the calculation of $P V_{d}$ no account is taken of the current rate of inflation.

The liability for a current pensioner is $M V_{p 1}(n)+M V_{p 2}(n)$ where

$$
\begin{aligned}
M V_{p 1}(n) & =\text { present value of the pension payments in the first } \\
& 12 \text { years using market rates of interest } \\
= & \ddot{a}_{65: n}, j
\end{aligned}
$$

where $j$ is calculated along the following lines:

- $\quad$ fixed pension increases of $p$ per annum $\Rightarrow j=\left(y_{0}-p\right) /(1+p)$ and $\left(y_{0}\right.$ is the annualised yield on the 15-year medium-coupon gilts index;

- $\quad$ RPI increases $\Rightarrow j=y_{1}$ is equal to the annualised real yield on the over-5-year index-linked gilts index;

- $\quad$ LPI increases

$$
\Rightarrow j=\max \left\{\frac{y_{0}-0.05}{1.05}, y_{1}+0.005\right\}
$$

The first value of $j$ allows the valuation of an annuity increasing at 5\% per annum which gives an upper bound to the cost. The second value values a pension which increases in line with RPI minus $\frac{1}{2} \%$ which is intended as a best estimate of the cost and not as an upper bound.

We also have

$$
\begin{aligned}
P V_{p 2}(n) & =v_{i=i^{\prime}}^{n} \times{ }_{n} p_{x} \times \ddot{a}_{x+n, i^{\prime \prime}} \\
M V_{p 2}(n) & =P V_{p 2}(n) \times M V A_{e q} \\
i^{\prime \prime} & =1.1 / 1.035-1=0.06280
\end{aligned}
$$

Finally we must determine the value of $n$. In general $n$ should be equal to 12 for a large scheme and $\infty$ for a small scheme. More precisely it can be calculated along the following lines:

$$
n= \begin{cases}\infty & \text { if } M V_{a}+M V_{d}<100 \text { Million } \\ \min \left\{m: m \in \mathbf{Z} ; M V_{p 1}(m) \geq 100 M\right\} & \text { if } M V_{a}+M V_{d} \geq 100 \text { Million }\end{cases}
$$


In this analysis no attempt was made to calculate $n$ with the level of accuracy dictated above as pensions were run off. Instead we took $n=12$ and $n=40$ to allow comparison of very large with very small schemes. The emphasis is therefore on identifying the differences between a gilt/equity strategy and a pure-gilt strategy.

No allowance was made for the costs of winding up. This is essentially independent of the question being addressed in this paper. 


\section{APPENDIX B \\ ACTIVES SIMULATIONS}

In the main text it was noted that there were 10,000 dependent simulations for active members. More precisely one very long simulation run of the Wilkie model was carried out (just over 10,000 years). A member aged 55 (or 25) at exit was assumed to leave the scheme with an un-revalued deferred pension of 1 per annum at the beginning of each year (year 1 up to $10,000)$ in this long simulation run. Each member was followed through to retirement. Starting from time $t(t=1, \ldots, 10000)$ we use the simulated investment returns on equities and gilts in an appropriate way over the period $t$ up to $t+(65-x)$ to calculate the accumulated fund at $t+65-x$. This is then divided by the simulated annuity rate at time $t+65-x$ to give the amount of the PP. The actual DP is equal to the unrevalued DP multiplied by the minimum of $R P I(t+65-x) / R P I(t)$ and $1.05^{65-x}$. This gives 10,000 realisations of the ratio $P P / D P$ from random, rather than neutral, starting points which we denote here by $\rho_{1}, \ldots, \rho_{10000}$. Clearly there is some dependence between sucessive observations, so that this sample is not as accurate as having 10,000 independent observations which would require over 100,000 (400,000) years' worth of simulation of the Wilkie model which is very time consuming. On the other hand, given that we have 10,000 years' worth of Wilkie simulation the method used here does make the most effective use of the available data. 


\section{APPENDIX C PENSIONERS}

The model pensioner membership ( 25 members) was as follows:

$\begin{array}{crr}\text { Pensioner } & \text { Current } & \text { Current } \\ 1 & \text { age } & \text { pension }(£) \\ 2 & 65 & 47,856 \\ 3 & 65 & 65,767 \\ 4 & 65 & 24,747 \\ 5 & 65 & 14,127 \\ 6 & 65 & 9,315 \\ 7 & 65 & 26,204 \\ 8 & 65 & 11,488 \\ 9 & 65 & 47,531 \\ 10 & 65 & 29,996 \\ 11 & 65 & 29,463 \\ 12 & 65 & 1,845 \\ 13 & 65 & 14,722 \\ 14 & 65 & 43,990 \\ 15 & 69 & 35,540 \\ 16 & 69 & 12,384 \\ 17 & 69 & 14,353 \\ 18 & 69 & 46,133 \\ 19 & 69 & 3,944 \\ 20 & 73 & 23,245 \\ 21 & 73 & 5,560 \\ 22 & 73 & 6,666 \\ 23 & 73 & 20,194 \\ 24 & 77 & 49,750 \\ 25 & 77 & 58,142 \\ 56 h+17 & 16,104\end{array}$

The scheme which has 100 members had four pensioners identical to each of the 25 above with each of the four having independent mortality. 